\title{
CLUMP-3D: the lack of non-thermal motions in galaxy cluster cores
}

\author{
Jack Sayers ${ }^{\circledR},{ }^{1 \star}$ Mauro Sereno ${ }^{\circledR},{ }^{2,3}$ Stefano Ettori ${ }^{\circledR},{ }^{2,3}$ Elena Rasia ${ }^{\circledR}$, 4 Weiguang Cui ${ }^{\circledR}, 5$ \\ Sunil Golwala, ${ }^{1}$ Keiichi Umetsu ${ }^{\circ 6}$ and Gustavo Yepes ${ }^{7,8}$ \\ ${ }^{1}$ California Institute of Technology, 1200 East California Boulevard, Pasadena, California 91125, USA \\ ${ }^{2}$ Osservatorio di Astrofisica e Scienza dello Spazio di Bologna, via Piero Gobetti 93/3, I-40129 Bologna, Italy \\ ${ }^{3}$ INFN, Sezione di Bologna, viale Berti Pichat 6/2, I-40127 Bologna, Italy \\ ${ }^{4}$ INAF - Osservatorio Astronomico di Trieste, via Tiepolo 11, I-34143 Trieste, Italy \\ ${ }^{5}$ Institute for Astronomy, University of Edinburgh, Royal Observatory, Edinburgh EH9 3HJ, UK \\ ${ }^{6}$ Academia Sinica Institute of Astronomy and Astrophysics (ASIAA), No. 1, Section 4, Roosevelt Road, Taipei 10617, Taiwan \\ ${ }^{7}$ Departamento de Física Teórica M-8, Universidad Autónoma de Madrid, Cantoblanco, E-28049 Madrid, Spain \\ ${ }^{8}$ Centro de Investigación Avanzada en Física Fundamental (CIAFF), Universidad Autónoma de Madrid, E-28049 Madrid, Spain
}

Accepted 2021 May 22. Received 2021 May 21; in original form 2021 March 5

\begin{abstract}
We report the non-thermal pressure fraction $\left(P_{\mathrm{nt}} / P_{\text {tot }}\right)$ obtained from a three-dimensional triaxial analysis of 16 galaxy clusters in the CLASH sample using gravitational lensing (GL) data primarily from Subaru and HST, X-ray spectroscopic imaging from Chandra, and Sunyaev-Zel'dovich effect (SZE) data from Planck and Bolocam. Our results span the approximate radial range $0.015-0.4 \mathrm{R}_{200 \mathrm{~m}}(\sim 35-1000 \mathrm{kpc})$. At cluster-centric radii smaller than $0.1 \mathrm{R}_{200 \mathrm{~m}}$ the ensemble average $P_{\mathrm{nt}} / P_{\text {tot }}$ is consistent with zero with an upper limit of 9 per cent, indicating that heating from active galactic nuclei and other relevant processes does not produce significant deviations from hydrostatic equilibrium (HSE). The ensemble average $P_{\mathrm{nt}} / P_{\text {tot }}$ increases outside of this radius to approximately 20 per cent at $0.4 \mathrm{R}_{200 \mathrm{~m}}$, as expected from simulations, due to newly accreted material thermalizing via a series of shocks. Also in agreement with simulations, we find significant cluster-to-cluster variation in $P_{\mathrm{nt}} / P_{\text {tot }}$ and little difference in the ensemble average $P_{\mathrm{nt}} / P_{\text {tot }}$ based on dynamical state. We conclude that on average, even for diverse samples, HSE-derived masses in the very central regions of galaxy clusters require only modest corrections due to non-thermal motions.
\end{abstract}

Key words: gravitational lensing: strong-gravitational lensing: weak-galaxies: active-galaxies: clusters: general-galaxies: clusters: intracluster medium-X-rays: galaxies: clusters.

\section{INTRODUCTION}

Given the hierarchical buildup of structure in the Universe, galaxy clusters are the largest and most recent objects to form (Davis et al. 1985; Kravtsov \& Borgani 2012). Their evolution is dictated mainly by gravity (Kaiser 1986, 1991), but a range of more complicated and less understood processes also play a significant role (e.g. Brunetti \& Lazarian 2007; Markevitch \& Vikhlinin 2007; McNamara \& Nulsen 2007). As a result, the diffuse ionized gas of the intra-cluster medium (ICM), which contains most of the baryons, does not reside in strict hydrostatic equilibrium (HSE) with the gravitational potential (Pratt et al. 2019; Ansarifard et al. 2020).

For instance, radiative cooling is capable of producing large reservoirs of cold star-forming gas in the central regions, but such reservoirs are not observed (Peterson \& Fabian 2006). While the cooling may be offset and/or suppressed by a range of processes, including core sloshing (Markevitch \& Vikhlinin 2007), dynamical friction from galaxy motions (El-Zant, Kim \& Kamionkowski 2004), and conduction and turbulent mixing (Ruszkowski \& Oh 2010), feedback from centrally located active galactic nuclei (AGNs) is thought to be the primary heating source (McNamara \& Nulsen 2007; Gitti, Brighenti \& McNamara 2012). This feedback is dynamic:

^E-mail: jack@caltech.edu
AGN jets inflate bubbles in the gas and transfer energy via multiple mechanisms, such as shocks, cavity heating, and convective mixing (Yang \& Reynolds 2016).

Recently, Hitomi used high-resolution X-ray spectroscopy to measure the gas velocity structure of Perseus's core. Surprisingly, despite its active AGN and the presence of features like a sloshing cold front (Simionescu et al. 2012), the gas was found to be remarkably quiescent, with a non-thermal pressure fraction $P_{\mathrm{nt}} / P_{\text {tot }} \simeq 4$ per cent (Hitomi Collaboration et al. 2016). More recently, a multiprobe observational study of five galaxy clusters at $z \simeq 0.35$ with extremely round morphologies from the Joint Analysis of Cluster Observations (JACO) project found $P_{\mathrm{nt}} / P_{\mathrm{tot}} \leqslant 6$ per cent within $R_{2500 \mathrm{c}}$ (corresponding to $\sim 0.2 \mathrm{R}_{200 \mathrm{~m}}$, Siegel et al. 2018). ${ }^{1}$ This suggests that Perseus is not unusual, although the strict selection of the JACO sample may not be representative of the overall population.

External to these central regions, simulations predict that $P_{\mathrm{nt}} / P_{\text {tot }}$ increases with radius as a result of the series of shocks required to thermalize newly accreted material (Miniati et al. 2000; Molnar,

\footnotetext{
${ }^{1}$ We refer to radii, and corresponding masses, that enclose an average density defined by the subscript. The number in the subscript denotes the overdensity relative to the background critical (c) or matter (m) density of the universe. All physical values described in this work were computed assuming a flat $\Lambda \mathrm{CDM}$ cosmology with $\Omega_{\mathrm{m}}=0.3, \Omega_{\Lambda}=0.7$, and $h=0.7$.
} 
Hearn \& Stadel 2012; Nelson, Lau \& Nagai 2014; Shi \& Komatsu 2014; Lau et al. 2015; Shi et al. 2015). Recently, JACO (Siegel et al. 2018) and the XMM-Newton Cluster Outskirts Project (XCOP; Eckert et al. 2019), which included 12 local, mostly relaxed, massive galaxy clusters, both found $P_{\mathrm{nt}} / P_{\text {tot }} \lesssim 10$ per cent at $R_{500 \mathrm{c}}$ $\left(\sim 0.5 \mathrm{R}_{200 \mathrm{~m}}\right)$. These results are in some tension with simulationbased predictions of 15-25 per cent at this radius (Nelson et al. 2014; Angelinelli et al. 2020; Gianfagna et al. 2020), and potentially imply more efficient thermalization.

The result presented here is obtained from modelling multiprobe observations using the CLUMP-3D (CLUster Multi Probe-3 Dimensions) package (Sereno et al. 2017). The available multiwavelength data enable independent measurements of the thermal pressure $\left(P_{\text {th }}\right)$ and total mass distribution (which sets the total pressure, $P_{\text {tot }}$, required to offset gravity). The non-thermal pressure $\left(P_{\mathrm{nt}}\right)$ is the difference between $P_{\text {tot }}$ and $P_{\text {th }}$. While this basic formalism is common to CLUMP-3D, JACO, and X-COP, our model relies on fewer assumptions. First and foremost, we do not use a spherically symmetric model, which can significantly bias the results from multiprobe reconstructions due to the strong degeneracy between the line-of-sight extent of the galaxy cluster and the inferred nonthermal pressure fraction (e.g. see the discussion in Section 7.2 of Siegel et al. 2018). In addition, we also allow for intrinsic clusterto-cluster scatter in $P_{\mathrm{nt}} / P_{\text {tot }}$. Unlike JACO, we do not assume a fixed radial form for $P_{\mathrm{nt}} / P_{\mathrm{tot}}$. Unlike X-COP, we measure the total mass with gravitational lensing (GL). Like JACO, we use strong lensing (SL) constraints to reliably probe mass profiles near the core. In sum, our technique enables a more flexible, data-driven approach that can address larger and more diverse samples.

\section{DATA}

Because of the deep multiprobe observations required for this type of analysis, previous applications of similar methods have been limited to individual galaxy clusters (e.g. Morandi et al. 2012; Limousin et al. 2013; Sereno et al. 2013). In this work, we use the data sets obtained as part of the Multi-Cycle Treasury program Cluster Lensing and Supernova survey with Hubble (CLASH; Postman et al. 2012) to model 16 individual objects. While CLASH includes 25 galaxy clusters, five were selected based on lensing strength and appear to be dynamically complicated systems that may not be accurately modelled within the CLUMP-3D formalism (i.e. at least four of these five objects are undergoing major mergers between at least two distinct sub-clusters; see Mann \& Ebeling 2012; Postman et al. 2012). Of the remaining 20, four lack the requisite ground-based wide-field GL data required for our analysis (Umetsu et al. 2018), leaving a sample of 16 for this work (see Table 1).

All of these clusters have a regular X-ray morphology, which indicates a higher than average probability of being dynamically relaxed (Meneghetti et al. 2014). None of the galaxy clusters appear to be undergoing a major merger. However, 8 of the 16 show potential signs of some merger activity in at least one systematic search for such objects based on X-ray imaging, location of the central galaxy, and/or member-galaxy velocity dispersions (Gilmour, Best \& Almaini 2009; Mann \& Ebeling 2012; Postman et al. 2012)

Observational data available for the full sample include: X-ray spectroscopic imaging from Chandra with a median exposure time of $44 \mathrm{ksec}$ (Sereno et al. 2018); Sunyaev-Zel'dovich effect (SZE) imaging from Bolocam and Planck with a median combined signalto-noise ratio of 12.3 (Sayers et al. 2016); wide-field ground-based weak lensing (WL) constraints from an average background source density of 12 arcmin $^{-2}$ after stringent colour-colour cuts (mainly
Table 1. Galaxy cluster sample. $R_{200 \mathrm{~m}}$ (and $M_{200 \mathrm{~m}}$ ) corresponds to the spherical volume determined from our fits to the CLUMP-3D model (Sereno et al. 2018). $R_{\max }$ is the maximum radius probed by the Chandra observations. While all of the objects have a regular X-ray morphology, which suggests they are more likely than average to be dynamically relaxed, those with any evidence of potential merger activity are indicated by stars (Gilmour et al. 2009; Mann \& Ebeling 2012; Postman et al. 2012; Meneghetti et al. 2014)

\begin{tabular}{lrrcc}
\hline Cluster Name & Redshift & $\begin{array}{c}M_{200 \mathrm{~m}} \\
\left(10^{14} \mathrm{M}_{\odot}\right)\end{array}$ & $\begin{array}{c}R_{200 \mathrm{~m}} \\
(\mathrm{Mpc})\end{array}$ & $\begin{array}{c}R_{\max } \\
\left(\mathrm{R}_{200 \mathrm{~m}}\right)\end{array}$ \\
\hline ABELL 0383 & 0.188 & $8.0 \pm 1.6$ & $2.41 \pm 0.16$ & 0.39 \\
ABELL 0209* & 0.206 & $11.4 \pm 4.0$ & $2.67 \pm 0.31$ & 0.51 \\
ABELL 2261* & 0.225 & $23.3 \pm 4.0$ & $3.36 \pm 0.19$ & 0.32 \\
RX J2129.6+0005* & 0.234 & $7.0 \pm 1.9$ & $2.22 \pm 0.20$ & 0.62 \\
ABELL 0611 & 0.288 & $8.9 \pm 2.1$ & $2.30 \pm 0.19$ & 0.31 \\
MACS J2140.2-2339 & 0.313 & $9.4 \pm 2.9$ & $2.30 \pm 0.19$ & 0.22 \\
ABELL S1063* & 0.348 & $16.6 \pm 3.7$ & $2.71 \pm 0.20$ & 0.50 \\
MACS J1115.8+0129 & 0.352 & $18.9 \pm 3.9$ & $2.82 \pm 0.19$ & 0.55 \\
MACS J1931.8-2635 & 0.352 & $7.6 \pm 2.0$ & $2.08 \pm 0.18$ & 0.68 \\
MACS J1532.8+3021 & 0.363 & $7.3 \pm 1.9$ & $2.03 \pm 0.17$ & 0.39 \\
MACS J1720.2+3536 & 0.391 & $10.4 \pm 2.4$ & $2.25 \pm 0.17$ & 0.45 \\
MACS J0429.6-0253 & 0.399 & $7.3 \pm 1.4$ & $1.98 \pm 0.13$ & 0.27 \\
MACS J1206.2-0847* & 0.440 & $17.1 \pm 2.1$ & $2.56 \pm 0.11$ & 0.41 \\
MACS J0329.6-0211* & 0.450 & $11.9 \pm 2.9$ & $2.25 \pm 0.14$ & 0.28 \\
MACS J1347.5-1144* & 0.451 & $35.0 \pm 6.6$ & $3.23 \pm 0.20$ & 0.60 \\
MACS J0744.9+3927* & 0.686 & $17.6 \pm 5.4$ & $2.21 \pm 0.28$ & 0.54 \\
\hline
\end{tabular}

using Subaru imaging from $\geq 3$ bands; Umetsu et al. 2014, 2018); and 16-filter HST imaging providing a median of 18 effective SL constraints per galaxy cluster and an average background source density of $50 \mathrm{arcmin}^{-2}$ for WL (Zitrin et al. 2015).

\section{METHODS}

\subsection{Three-dimensional model}

The CLUMP-3D model assumes an elliptical triaxial geometry, with co-alignment and co-centering of the major, intermediate, and minor axes of both the total mass and gas distributions. The eccentricities are allowed to separately vary, with the well-motivated prior that the gas distribution is rounder (Lau et al. 2011). As a function of the elliptical radial coordinate, the total mass density is parametrized by the Navarro-Frenk-White (NFW) profile (Navarro, Frenk \& White 1996), while the gas density and temperature are parametrized by a modified beta-model and a modified broken power law (Vikhlinin et al. 2006). The model has seven free parameters related to the shape and orientation of the galaxy cluster: two axial ratios for both the total mass density and the gas density, along with three angles to describe the orientation in the observer's reference frame. In addition, the total mass density has two free parameters, the gas density has six free parameters, and the gas temperature has five free parameters.

Within this model, X-ray, and SZE data mostly constrain the parameters related to the gas, while the GL data constrain the parameters related to the total mass. Specifically, the X-ray surface brightness is proportional to $\int \rho_{\text {gas }}^{2} \Lambda \mathrm{d} l$, where $\rho_{\text {gas }}$ is the gas density, $\Lambda$ is the X-ray cooling function, and $\mathrm{d} l$ is along the line of sight through the galaxy cluster. Independently, the gas temperature $\left(T_{\text {gas }}\right)$ can be determined from X-ray spectroscopy. The SZE brightness is proportional to $\int P_{\mathrm{th}} \mathrm{d} l$, where $P_{\text {th }} \propto \rho_{\text {gas }} T_{\text {gas }}$. Because the X-ray and SZE data redundantly probe the value of $P_{\text {th }}$, but with a different dependence on the integral $\mathrm{d} l$, their combination measures the lineof-sight extent of the galaxy cluster and thus its three-dimensional 


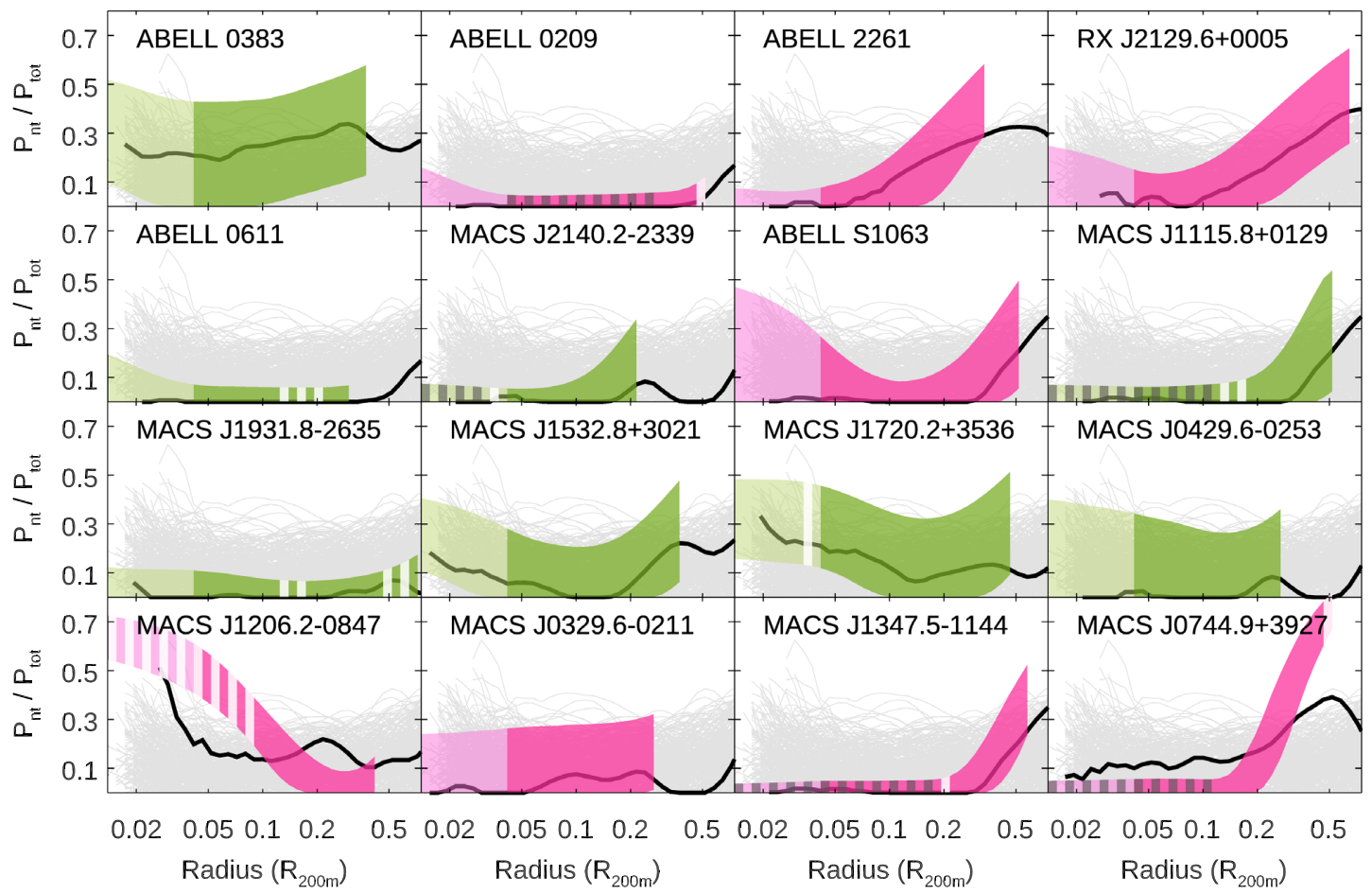

Figure 1. The shaded bands indicate 95 per cent confidence regions of $P_{\mathrm{nt}} / P_{\text {tot }}$ for each galaxy cluster, restricted to physically allowed values $\geq 0$ using the technique of Feldman \& Cousins (1998). Pink denotes objects with potential merger activity and green denotes non-mergers (Gilmour et al. 2009; Mann \& Ebeling 2012; Postman et al. 2012; Meneghetti et al. 2014). Potential systematic errors in our modelling may result in unaccounted for biases at $R \leq 0.04 \mathrm{R}_{200 \mathrm{~m}}$, which is denoted with a lighter colour. Dark grey hashes indicate values of $P_{\mathrm{nt}} / P_{\text {tot }}$ removed from the analysis based on goodness-of-fit to the physically allowed region and white hashes indicate values of $P_{\mathrm{nt}} / P_{\text {tot }}$ removed from the analysis based on goodness-of-fit to the ensemble average profile (see text for additional details). The profiles for 315 galaxy clusters from the 300 simulations (Cui et al. 2018) are shown as thin grey lines, with the best match to the observed profile highlighted as a thicker black line.

geometry. The GL data directly probe the projected mass density, which can then be used to measure the three-dimensional total mass distribution based on the geometry which is primarily determined from the X-ray and SZE data.

From the observational data available, the following products are generated to constrain the model using the brightest cluster galaxy as the centre (Sereno et al. 2017, 2018). Background and exposurecorrected Chandra surface brightness images are produced in the $0.7-2.0 \mathrm{keV}$ band using the CIAO 4.8 software and the calibration data base CALDB 4.7.1. Point sources are filtered out, and the two-dimensional image is restricted to the circular region enclosing 80 per cent of the total source emission. Spectra used to constrain the gas temperature are extracted from circular annuli and analysed with the XSPEC v.12.9 software. The SZE brightness is computed in circular annuli using publicly available maps from Bolocam (Sayers et al. 2013) and Planck (Planck Collaboration XXII 2016) via a combined analysis of both data sets (Sayers et al. 2016). For the ground-based wide-field WL, two-dimensional projected mass maps and their pixel-pixel covariance matrices are obtained from a joint analysis of the shear and magnification bias over a $24 \times 24 \mathrm{arcmin}^{2}$ square region (Umetsu et al. 2018). Using the Hubble Space Telescope WL and SL data, projected masses are computed in circular annuli between 5 arcsec and twice the Einstein radius from the publicly available CLASH PIEMDeNFW maps (Zitrin et al. 2015).

To compare with these products generated from the observational data, the CLUMP-3D model is used to create projected GL mass maps, X-ray surface brightness, and temperature maps, and SZE brightness maps from a given set of parameter values. A Bayesian inference scheme is used to assess the probability distributions of the parameters (Sereno et al. 2017). Priors spanning large regions of parameter space, with uniform distributions in either linear or logarithmic spaced intervals, are assumed. Some priors span the full range of physically motivated or allowed values. For example, the major axial ratio of the total mass density has a uniform linear prior between 0.1 and 1.0 (Jing \& Suto 2002). All other priors span a sufficiently large range to include any reasonable derived value.

\subsection{Non-thermal pressure analysis}

From these fits, we compute probability densities for the values of $\rho_{\text {gas }}$ and the gravitational potential $\Phi_{\text {mat }}$ in three dimensions. Here, we assume the gas to be in equilibrium, and so the ICM axial ratios are set equal to the average ratios of the gravitational potential. We then determine $P_{\text {tot }}$ from the HSE equation $\left(\nabla P_{\text {tot }}=-\rho_{\text {gas }} \nabla \Phi_{\text {mat }}\right)$. Using the three-dimensional values for $P_{\mathrm{th}}$ and $P_{\mathrm{tot}}$, we compute the average for each within a set of discrete logarithmically spaced spherical annuli. We find that the probability densities are well described by a Gaussian distribution for $\ln \left(P_{\mathrm{th}} / P_{\mathrm{tot}}\right)$, and so profiles are generated in this quantity for each galaxy cluster. Finally, the radial coordinate is scaled according to $\mathcal{R}=\mathrm{R} / \mathrm{R}_{200 \mathrm{~m}}$, which simulations indicate minimizes the cluster-to-cluster scatter in $P_{\mathrm{nt}} / P_{\text {tot }}$ (Nelson et al. 2014). Plots of $P_{\mathrm{nt}} / P_{\text {tot }}$ for all 16 galaxy clusters, which is computed from the values of $\ln \left(P_{\mathrm{th}} / P_{\mathrm{tot}}\right)$ according to $P_{\mathrm{nt}}=P_{\mathrm{tot}}-P_{\mathrm{th}}$, are shown in Fig. 1. 
From these radially scaled profiles, we constrain the ensembleaverage profile at each discrete radius using the log likelihood

$\mathcal{L}\left(X \mid \mu, \sigma_{\text {int }}\right)=-\frac{1}{2} \sum_{i}^{N}\left[\frac{\left(x_{i}-\mu\right)^{2}}{\sigma_{i}^{2}+\sigma_{\text {int }}^{2}}+\ln \left(\sigma_{i}^{2}+\sigma_{\text {int }}^{2}\right)+\ln (2 \pi)\right]$,

where $X_{\mathrm{m}}=\left\{x_{1}, \ldots, x_{N}\right\}$ are the values of $\ln \left(P_{\mathrm{th}} / P_{\mathrm{tot}}\right)$ with measurement uncertainties $\sigma_{i}$ for the $N$ objects in our sample, $\mu$ is the ensemble average, and $\sigma_{\text {int }}$ is the intrinsic scatter about the average. This likelihood is evaluated using a grid of $\mu \in[-2,2]$ and $\sigma_{\text {int }} \in$ $[0,1]$ to obtain probability distributions for $\mu$ and $\sigma_{\text {int }}$. Note that the grid ranges are sufficiently large to include all values of $\mu$ and $\sigma_{\text {int }}^{2}$ with non-negligible likelihood values.

To mitigate the impact of outlier values on our ensemble analysis, we discard any $x_{i}$ that fails the goodness-of-fit criteria suggested by Feldman \& Cousins (1998). To ensure consistency with the physically allowed region based on this procedure, any individual $x_{i}$ with

$\int_{x_{i}}^{\infty} \mathcal{L}\left(x \mid \mu=0, \sigma_{\text {int }}=0\right) \mathrm{d} x<0.01$,

is removed from the analysis. This occurs at a subset of radii for five galaxy clusters, primarily at $\mathcal{R} \lesssim 0.1 \mathrm{R}_{200 \mathrm{~m}}$ (see Figs 1 and 2 ). As detailed in Section 4, our assumption of coalignment between the gas density and the gravitational potential may be invalid at $\mathcal{R} \lesssim$ $0.04 \mathrm{R}_{200 \mathrm{~m}}$. We therefore suspect biases related to the breakdown of this assumption are the underlying cause of the un-physical values seen in these five clusters, which provides additional motivation for removing them from the ensemble analysis.

To further minimize contamination from outliers, we also remove any $x_{i}$ that sufficiently differs from the maximum likelihood value of $\mu$ such that they would be expected to occur less than 1 per cent of the time given the maximum likelihood value of $\sigma_{\text {int }}$. This removal is performed in an iterative manner until no such $x_{i}$ remain, and primarily impacts one object, MACS J1206.2-0847, at $\mathcal{R} \lesssim 0.1 R_{200 \mathrm{~m}}$, where it has a much larger non-thermal pressure fraction than any other galaxy cluster in our sample. As with the un-physical $P_{\mathrm{nt}} / P_{\text {tot }}$ values noted above, we suspect these values are also due to a breakdown of our assumption of coalignment between the gas density and the gravitational potential. In addition to MACS J1206.2-0847, data are also removed from other objects based on this criteria. However, in those instances the removal is limited to a narrow radial range (see Figs 1 and 2).

From a total of 16 values, one for each galaxy cluster, the number of $x_{i}$ eliminated at a given radius due to the combination of both removal processes detailed above is between 1 and 6 , and so the majority of the sample is retained for the ensemble fits. The value of $\mu$ is largely insensitive to these data cuts, ${ }^{2}$ even if no $x_{i}$ are removed. However, the value of $\sigma_{\text {int }}$ does increase if fewer $x_{i}$ are removed, particularly at small radii where it can be up to a factor of $\simeq 1.5$ larger when all the $x_{i}$ are retained.

\subsection{Confidence intervals in the physically allowed region}

We evaluate the likelihood at $\mu>0$, which is in the physically disallowed region corresponding to $P_{\mathrm{th}}>P_{\mathrm{tot}}$ (i.e. $\left.P_{\mathrm{nt}}<0\right)$. Establishing

\footnotetext{
${ }^{2}$ It may appear that the data cuts will shift the ensemble mean $P_{\mathrm{nt}} / P_{\text {tot }}$ to lower values at small radii due to the exclusion of data from MACS J1206.2-0847 (see Fig. 1). However, in aggregate, the data cuts actually result in slightly higher values for the ensemble mean $P_{\mathrm{nt}} / P_{\text {tot }}$ at those radii, since data are also excluded from other galaxy clusters with very low values of $P_{\mathrm{nt}} / P_{\mathrm{tot}}$.
}

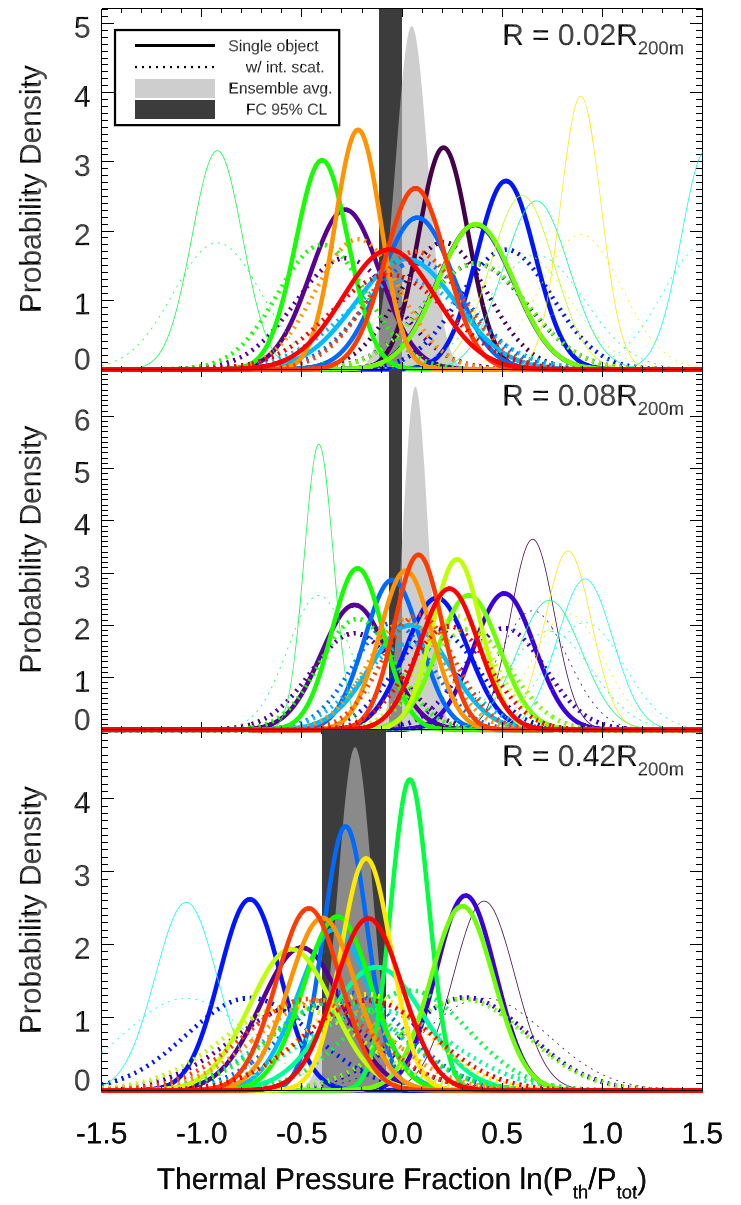

Figure 2. Measured probability densities for the logarithmic thermal pressure fraction $\ln \left(P_{\mathrm{th}} / P_{\mathrm{tot}}\right)$ at three different radii. Each solid line represents a single cluster when considering only the statistical uncertainties. The dashed lines indicate the effective broadening of the distributions when the fitted intrinsic scatter is included. The shaded grey region indicates the distribution for the ensemble average, including intrinsic scatter. Solid black encloses the 95 per cent confidence region within the physically allowed set of values based on the Feldman \& Cousins (1998) technique (i.e. $\ln \left(P_{\text {th }} / P_{\text {tot }}\right) \leq 0$ ). Thinner lines represent galaxy clusters that were excluded from the ensemble analysis based on the criteria described in the text.

confidence intervals on the values of $\mu$ requires care due to this physical boundary. For instance, imposing the physical boundary on the $x_{i}$ prior to computing $\mu$ can result in biases (Leccardi \& Molendi 2008). Furthermore, Feldman \& Cousins (1998) have argued that simple prescriptions such as Bayesian priors or renormalization of the Bayesian posterior can produce intervals without the desired coverage. For this analysis, we adopt the Feldman \& Cousins (1998) approach for establishing frequentist confidence intervals in the physically allowed region.

Specifically, a value of $\mu$ should be included in the $\alpha$ per cent confidence interval if the data $X_{\mathrm{m}}$ are in the $\alpha$ per cent most likely observational outcomes were $\mu$ the true underlying value. As described by Feldman \& Cousins (1998), the statistic for evaluating whether an outcome is in the $\alpha$ per cent most likely outcomes is

$R(X)=\frac{\mathcal{L}(X \mid \mu)}{\mathcal{L}\left(X \mid \mu^{\star}\right)}$

where $\mu^{\star}$ maximizes the likelihood within the physically allowed region (i.e. $\left.\mu^{\star} \leq 0\right)$ and $\mathcal{L}(X \mid \mu)$ is obtained from equation (1). 
Absent a physical boundary, the denominator is independent of $X$ and $R(X) \propto \mathcal{L}(X \mid \mu)$. Because every potential observational outcome $X$ yields a value $R(X), \mathcal{L}(X \mid \mu)$ maps into a likelihood $\mathcal{L}^{\prime}(R \mid \mu)$. We define a value $R_{\alpha}$ such that the integral of $\mathcal{L}^{\prime}(R \mid \mu)$ over $R(X) \geq R_{\alpha}$ is equal to $\alpha$. We include a value of $\mu$ in the $\alpha$ per cent confidence interval if $R\left(X_{\mathrm{m}} \mid \mu\right) \geq R_{\alpha}$. To obtain the complete desired confidence interval, this process is performed over a grid of values of $\mu$.

To assess the validity of our measured values of $\mu$ given the physical boundary $\mu \leq 0$, we again consider the Feldman \& Cousins (1998) goodness-of-fit test described above and quantified in equation (2) for the case of the individual $x_{i}$. We perform an analogous test for the values of $\mu$ obtained in our grid search at each radius. The worst goodness-of-fit values occur at intermediate radii from $\sim 0.05-0.09 \mathrm{R}_{200 \mathrm{~m}}$, and correspond to a probability to exceed of approximately $0.70-0.80$ (e.g. see the middle panel of Fig. 2). This goodness-of-fit is significantly better than the threshold suggested by Feldman \& Cousins (1998), corresponding to a probability to exceed of 0.99 , and suggests that none of our measured $\mu$ fall sufficiently outside of the physically allowed region to be considered invalid.

\subsection{Analysis of simulated galaxy clusters}

We apply the same analysis to $P_{\text {th }}$ and $P_{\text {tot }}$ profiles obtained in spherical annuli from 315 simulated galaxy clusters in the Three Hundred Project (hereafter '300'; Cui et al. 2018). This sample was selected based on the criteria described in Ansarifard et al. (2020), and excludes nine objects with at least one low-resolution particle, which are used to trace the large-scale structure in which the galaxy cluster is embedded, within $R_{200 \mathrm{~m}}$. These haloes span the approximate mass range $M_{200 \mathrm{~m}} \simeq 10-25 \times 10^{14} \mathrm{M}_{\odot}$, nearly identical to that of the CLASH objects, and they were taken from a snapshot at $z=0.333$, close to the median $z$ of 0.352 for our observational sample.

\section{RESULTS AND DISCUSSION}

Using the procedure detailed in Section 3, we obtain 95 per cent confidence regions for $P_{\mathrm{nt}} / P_{\text {tot }}$ profiles for all 16 individual galaxy clusters (see Fig. 1), along with the ensemble average $P_{\mathrm{nt}} / P_{\text {tot }}$ and the intrinsic scatter about this average (see Figs 3 and 4). When interpreting these profiles, note that the CLUMP-3D model assumes coalignment of the gas and total mass over the entire radial range. While simulations indicate this assumption is likely valid outside of $0.1 \mathrm{R}_{500 \mathrm{c}}\left(\sim 0.04 \mathrm{R}_{200 \mathrm{~m}}\right.$; Lau et al. 2011$)$, it might not be true at smaller radii where non-gravitational processes may play a larger role (McNamara \& Nulsen 2007; Markevitch \& Vikhlinin 2007). In addition, modelling of the Chandra data is generally more prone to systematic errors within approximately $50 \mathrm{kpc}\left(\sim 0.02 \mathrm{R}_{200 \mathrm{~m}}\right)$ due to inhomogeneities in the gas distribution (McNamara \& Nulsen 2007). The Chandra data also set the maximum radius that is fully constrained by GL, X-ray, and SZE data, with $R_{\max }=0.22$ $0.68 \mathrm{R}_{200 \mathrm{~m}}$ (see Table 1 ). In our ensemble analysis, the maximum radius considered is $0.42 R_{200 m}$, outside of which more than half the sample lacks Chandra coverage.

Within $0.1 R_{200 m}$, we measure an ensemble-average non-thermal pressure fraction consistent with zero (see Fig. 3). The 95 per cent confidence level upper limit takes on values in the range 818 per cent, with a volume-averaged upper limit of 9 per cent. This is consistent with the Hitomi measurements of Perseus (Hitomi Collaboration et al. 2018) and the spherical JACO analysis of five galaxy clusters with extremely round morphologies (Siegel et al. 2018). Our analysis therefore suggests that those previous results are valid for a larger and more diverse sample. Recent simulations

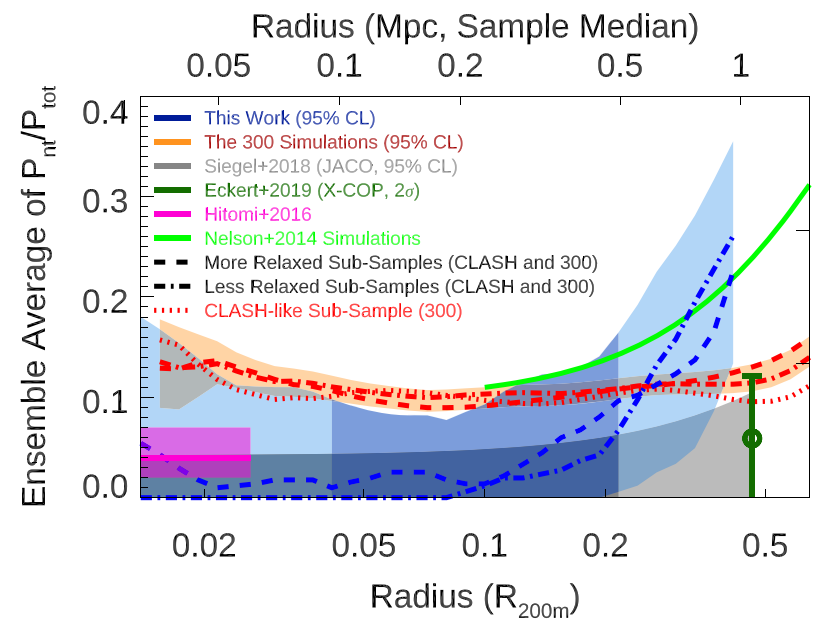

Figure 3. Ensemble-average $P_{\mathrm{nt}} / P_{\text {tot }}$ from our analysis of $16 \mathrm{CLASH}$ galaxy clusters (blue 95 per cent confidence region obtained from the Feldman \& Cousins 1998 procedure detailed in the text and corresponding to the solid black regions in Fig. 2) and the 300 simulations (orange 95 per cent confidence region; Cui et al. 2018). Lighter shading indicates regions where biases due to modelling systematics may exist. The widely used profile shape from Nelson et al. (2014) is shown as a green line. The Hitomi result for Perseus is shown in pink for the range of observed velocity dispersions (Hitomi Collaboration et al. 2018). Observational results from the multiprobe JACO and X-COP analyses are shown in grey (95 per confidence; Siegel et al. 2018) and green (2 $\sigma$; Eckert et al. 2019). Dashed and dot-dashed lines indicate the most likely profiles for the more relaxed and less relaxed sub-samples of the CLASH galaxy clusters and the 300 simulations. The red dotted line shows the most likely profile from a CLASH-like selection of 20 objects from the 300 simulations.

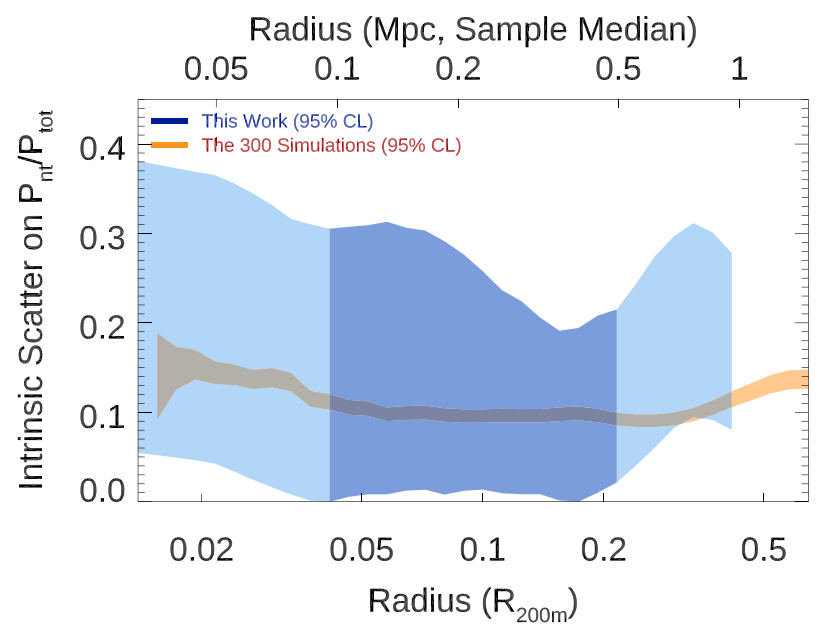

Figure 4. Intrinsic scatter on $P_{\mathrm{nt}} / P_{\text {tot }}$ (same convention as Fig. 3).

generally predict $P_{\mathrm{nt}} / P_{\text {tot }}$ values close to our measured upper limit (i.e. $\sim 10$ per cent, Angelinelli et al. 2020; Gianfagna et al. 2020), in good agreement with our analysis of the 300 simulations.

Beyond $0.1 \mathrm{R}_{200 \mathrm{~m}}$, our measured ensemble-average $P_{\mathrm{nt}} / P_{\text {tot }}$ increases. This is expected due to the series of shocks at varying radii that thermalize newly accreted material (Miniati et al. 2000; Molnar et al. 2012). Predictions from simulations (Nelson et al. 2014; Angelinelli et al. 2020; Gianfagna et al. 2020), including our analysis of the 300 simulations, fall within our measured 95 per cent confidence level region at these radii. The low values of $P_{\mathrm{nt}} / P_{\mathrm{tot}}$ obtained from the JACO and X-COP observational studies are in 
mild tension with our results, although this difference only appears at radii where some objects require an extrapolation beyond the Chandra data.

With a relatively large measurement uncertainty, the CLASH data indicate an intrinsic cluster-to-cluster scatter on $P_{\mathrm{nt}} / P_{\text {tot }}$ that is approximately constant with radius at a value of $15-20$ per cent. This scatter is generally comparable to, or larger than, the ensemble average value of $P_{\mathrm{nt}} / P_{\mathrm{tot}}$. Therefore, while the gas at small radii is highly quiescent on average, some galaxy clusters do contain significant non-thermal pressure support within that region. This suggests that AGN feedback may sometimes produce a larger amount of non-thermal pressure than typical and/or there are other relevant processes that destabilize the equilibrium near the cores of some galaxy clusters. Similarly, while the average value of $P_{\mathrm{nt}} / P_{\text {tot }}$ increases with radius, some galaxy clusters have very little nonthermal pressure at large radii. This likely reflects the range of possible accretion histories within the population (Shi \& Komatsu 2014). Our measured scatter is consistent with what is seen in the 300 simulations and other published simulations (Nelson et al. 2014; Shi et al. 2015; Angelinelli et al. 2020; Gianfagna et al. 2020), indicating that they accurately reproduce the level of observed diversity.

In addition, we compute $P_{\mathrm{nt}} / P_{\text {tot }}$ profiles for sub-samples of more relaxed and less relaxed galaxy clusters from both CLASH and the 300 simulations. For CLASH, the equal-sized sub-samples of eight objects are chosen based on the presence, or lack of, possible merger activity (Gilmour et al. 2009; Mann \& Ebeling 2012; Postman et al. 2012). For the 300 simulations, a sub-sample of 101 more relaxed systems was selected based on a centre-of-mass offset $\Delta_{\mathrm{r}} \leq 0.04$ and a fraction of mass in subhaloes $f_{\mathrm{s}} \leq 0.1$ within $\mathrm{R}_{500 \mathrm{c}}$ (Cui et al. 2018), with the remaining 214 objects forming the less relaxed subsample. These thresholds are commonly used, and are sufficient to identify objects undergoing relatively minor mergers (e.g. a single $10-1$ mass ratio sub-structure will generally produce a value of $f_{\mathrm{s}}$ above our cutoff). This criteria should be similar to that used to identify potential mergers in the CLASH objects. For instance, the lower limit on the mass of the sub-cluster involved in the minor merger ongoing in MACS J1347.5+1144 is sufficient to have a mass ratio of 10-1 (Johnson et al. 2012). Thus, we expect that the more relaxed sub-sample identified from the 300 simulation is comparable to that identified in the CLASH clusters. However, this is less likely to be true for the less relaxed sub-sample. As noted by Meneghetti et al. (2014), and further indicated by the larger fraction of less relaxed objects in the sub-sample selected from the 300 simulations ( 70 per cent) compared to CLASH (50 per cent), the CLASH selection excludes major mergers and extremely dynamically active systems (e.g. with evidence of multiple minor mergers) which are represented in the 300 simulations.

When considering the less relaxed and more relaxed sub-samples defined above, it is not possible to robustly constrain the intrinsic scatter with only eight objects in the CLASH sub-samples. Therefore, for these sub-sample fits, we fix the intrinsic scatter to the maximumlikelihood value obtained from the full sample. At all radii, the profiles of the more relaxed and less relaxed sub-samples are consistent for both the CLASH galaxy clusters and the 300 simulations, indicating that the ensemble average $P_{\mathrm{nt}} / P_{\text {tot }}$ is largely insensitive to dynamical state and thus not strongly influenced by merger activity, at least for non-major mergers. This conclusion is consistent with the findings of a separate analysis of the 300 simulations focused on mass calibration (Ansarifard et al. 2020), but in contrast to what has been found in some recent simulations (Nelson et al. 2014; Gianfagna et al. 2020).
Table 2. X-ray morphological parameters. Inter-quartile ranges are given for the CLASH sample (Donahue et al. 2016), a CLASH-like sub-sample of 20 objects selected to best match these parameters from the 80 most massive galaxy clusters in the 300 simulations, and the full set of 80 galaxy clusters.

\begin{tabular}{lccc}
\hline Parameter & CLASH & $300_{80}$ (CLASH-like) & $300_{80}$ (All) \\
\hline$C$ & $0.34-0.53$ & $0.42-0.54$ & $0.28-0.52$ \\
$w\left(10^{-2}\right)$ & $0.3-1.4$ & $0.4-1.5$ & $0.8-4.3$ \\
$A R$ & $0.87-0.95$ & $0.85-0.92$ & $0.72-0.87$ \\
$P 30\left(10^{-7}\right)$ & $0.4-1.6$ & $0.3-1.1$ & $0.2-8.5$ \\
\hline
\end{tabular}

While the average non-thermal pressure profile appears to be largely insensitive to dynamical state, the results described above do not definitively establish whether it is sensitive to the specific CLASH selection. Since the CLASH objects were selected primarily based on X-ray morphology, we follow the general approach of Meneghetti et al. (2014) to select a CLASH-like sample of galaxy clusters from the 300 simulations. Specifically, we consider the concentration $C$, centroid shift $w$, axial ratio $A R$, and power ratio $P 30$ determined for three orthogonal projections of the 80 most massive objects from the simulations (i.e. a total of 240 projections for the ' $300_{80}$ '). For each of the 16 CLASH galaxy clusters, we select the two projections that most closely match the values for these quantities measured by Donahue et al. (2016). This results in a sample of 20 unique galaxy clusters from the 300 simulations with X-ray morphologies closely matched to the CLASH galaxy clusters (see Table 2). Although there are possible hints of lower values for the average $P_{\mathrm{nt}} / P_{\text {tot }}$ computed from this CLASH-like sub-sample compared to the full sample, none of these differences are statistically significant given the size of the sub-sample (see Fig. 3). Furthermore, the absolute magnitude of these differences is relatively small (i.e. $\$ 3$ per cent). Thus, we conclude that the average non-thermal pressure profile obtained from the CLASH galaxy clusters is likely to be a good representation of the population as a whole, with no significant selection biases.

\section{SUMMARY AND CONCLUSIONS}

From a relatively diverse sample of 16 CLASH objects, we find that there is generally very little non-thermal pressure support in the core regions of galaxy clusters. Our result suggests that highly quiescent cores are not unusual nor restricted to a particular subset of galaxy clusters. This conclusion is further supported by the consistency of the non-thermal pressure fraction measured in two sub-samples of eight objects with and without potential merger activity. Therefore, AGN outbursts and other relevant heating mechanisms must typically operate in a gentle manner that preserves approximate HSE. Outside of the core, we find that the non-thermal pressure fraction increases with radius, as expected due to incomplete thermalization of newly accreted material. Furthermore, we find that the non-thermal pressure fraction varies significantly among the population, both within and external to the core region. Other than the 300 simulations suggesting a slightly higher ensemble average $P_{\mathrm{nt}} / P_{\text {tot }}$ near the galaxy clusters' centres, we find generally good agreement between the observational results from the CLASH sample and the results from those simulations. At the smaller radii, where indications of a possible difference do exist, our measured upper limit is similar to the average $P_{\mathrm{nt}} / P_{\text {tot }}$ found in the 300 simulations. Therefore, at a confidence level of approximately 95 per cent, our result is still consistent with the simulations in this radial range. This implies that our measurement is also consistent with the relatively small HSE-derived mass biases obtained from the 300 simulations at those radii (e.g. the 5-10 per 
cent bias found at $R_{2500 \mathrm{c}}$ by Ansarifard et al. 2020), suggesting that non-thermal motions in the central regions of galaxy clusters do not significantly impact HSE-derived masses.

\section{ACKNOWLEDGEMENTS}

The simulations of the 300 project were performed on the Marenostrum Supercomputer of the Barcelona Supercomputing Center thanks to computing time from the 'Red Española de Supercomputación'. JS was supported by NSF/AAG award 1617022. GY was supported by MICIU/FEDER (Spain) grant PGC2018-094975-C21. WC was supported by European Research Council grant 670193. KU was supported by the Ministry of Science and Technology of Taiwan (grants MOST 106-2628-M-001-003-MY3 and MOST 109-2112M-001-018-MY3) and from the Academia Sinica Investigator Award (grant AS-IA-107-M01). SE, ER, and MS were supported by contract ASI-INAF n.2017-14-H.0. SE and MS were supported by INAF mainstream project 1.05.01.86.10. SE was supported by contract ASI-INAF Athena 2019-27-HH.0, and by the European Union's Horizon 2020 Programme under the AHEAD2020 project (grant 871158).

\section{DATA AVAILABILITY}

The data underlying this analysis are available from repositories for CLASH https://archive.stsci.edu/prepds/clash/, Chandra https: //cxc.cfa.harvard.edu/cda/, and Planck https://irsa.ipac.caltech.edu/ Missions/planck.html.

\section{REFERENCES}

Angelinelli M., Vazza F., Giocoli C., Ettori S., Jones T. W., Brunetti G., Brüggen M., Eckert D., 2020, MNRAS, 495, 864

Ansarifard S. et al., 2020, A\&A, 634, A113

Brunetti G., Lazarian A., 2007, MNRAS, 378, 245

Cui W. et al., 2018, MNRAS, 480, 2898

Davis M., Efstathiou G., Frenk C. S., White S. D. M., 1985, ApJ, 292, 371

Donahue M. et al., 2016, ApJ, 819, 36

Eckert D. et al., 2019, A\&A, 621, A40

El-Zant A. A., Kim W.-T., Kamionkowski M., 2004, MNRAS, 354, 169

Feldman G. J., Cousins R. D., 1998, Phys. Rev. D, 57, 3873

Gianfagna G. et al., 2020, MNRAS , 502, 5113

Gilmour R., Best P., Almaini O., 2009, MNRAS, 392, 1509

Gitti M., Brighenti F., McNamara B. R., 2012, Adv. Astron., 2012, 950641

Hitomi Collaboration et al., 2016, Nature, 535, 117

Hitomi Collaboration et al., 2018, PASJ, 70, 9
Jing Y. P., Suto Y., 2002, ApJ, 574, 538

Johnson R. E., Zuhone J., Jones C., Forman W. R., Markevitch M., 2012, ApJ, 751, 95

Kaiser N., 1986, MNRAS, 222, 323

Kaiser N., 1991, ApJ, 383, 104

Kravtsov A. V., Borgani S., 2012, ARA\&A, 50, 353

Lau E. T., Nagai D., Kravtsov A. V., Zentner A. R., 2011, ApJ, 734, 93

Lau E. T., Nagai D., Avestruz C., Nelson K., Vikhlinin A., 2015, ApJ, 806, 68

Leccardi A., Molendi S., 2008, A\&A, 487, 461

Limousin M., Morandi A., Sereno M., Meneghetti M., Ettori S., Bartelmann M., Verdugo T., 2013, Space Sci. Rev., 177, 155

Mann A. W., Ebeling H., 2012, MNRAS, 420, 2120

Markevitch M., Vikhlinin A., 2007, Phys. Rep., 443, 1

McNamara B. R., Nulsen P. E. J., 2007, ARA\&A, 45, 117

Meneghetti M. et al., 2014, ApJ, 797, 34

Miniati F., Ryu D., Kang H., Jones T. W., Cen R., Ostriker J. P., 2000, ApJ, 542,608

Molnar S. M., Hearn N. C., Stadel J. G., 2012, ApJ, 748, 45

Morandi A. et al., 2012, MNRAS, 425, 2069

Navarro J. F., Frenk C. S., White S. D. M., 1996, ApJ, 462, 563

Nelson K., Lau E. T., Nagai D., 2014, ApJ, 792, 25

Peterson J. R., Fabian A. C., 2006, Phys. Rep., 427, 1

Planck Collaboration et al., 2016, A\&A, 594, A22

Postman M. et al., 2012, ApJS, 199, 25

Pratt G. W., Arnaud M., Biviano A., Eckert D., Ettori S., Nagai D., Okabe N., Reiprich T. H., 2019, Space Sci. Rev., 215, 25

Ruszkowski M., Oh S. P., 2010, ApJ, 713, 1332

Sayers J. et al., 2013, ApJ, 768, 177

Sayers J. et al., 2016, ApJ, 832, 26

Sereno M., Ettori S., Umetsu K., Baldi A., 2013, MNRAS, 428, 2241

Sereno M., Ettori S., Meneghetti M., Sayers J., Umetsu K., Merten J., Chiu I. N., Zitrin A., 2017, MNRAS, 467, 3801

Sereno M., Umetsu K., Ettori S., Sayers J., Chiu I. N., Meneghetti M., VegaFerrero J., Zitrin A., 2018, ApJ, 860, L4

Shi X., Komatsu E., 2014, MNRAS, 442, 521

Shi X., Komatsu E., Nelson K., Nagai D., 2015, MNRAS, 448, 1020

Siegel S. R. et al., 2018, ApJ, 861, 71

Simionescu A. et al., 2012, ApJ, 757, 182

Umetsu K. et al., 2014, ApJ, 795, 163

Umetsu K. et al., 2018, ApJ, 860, 104

Vikhlinin A., Kravtsov A., Forman W., Jones C., Markevitch M., Murray S.

S., Van Speybroeck L., 2006, ApJ, 640, 691

Yang H. Y. K., Reynolds C. S., 2016, ApJ, 829, 90

Zitrin A. et al., 2015, ApJ, 801, 44

This paper has been typeset from a $\mathrm{T}_{\mathrm{E}} \mathrm{X} / \mathrm{L} \mathrm{T}_{\mathrm{E}} \mathrm{X}$ file prepared by the author. 